\title{
Chromosome Condensation
}

National Cancer Institute

\section{Source}

National Cancer Institute. Chromosome Condensation. NCI Thesaurus. Code C19395.

A process of progressive and tight packing of dispersed replicated interphase chromatin into compact structures in metaphase prior to mitotic or meiotic nuclear division or during apoptosis in eukaryotic cells. 